Prof. Dr. med.

Thomas Lehrnbecher,

Frankfurt/Main

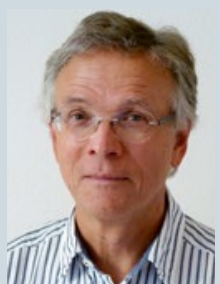

Dr. med.

Ulrich Mutschler,

Hamburg

\section{Alterskorrektur Frühgeborener bei der Intelligenzbeurteilung beachten}

\section{Alle Kinderärzte kennen die uralte Debatte: Soll die Beurteilung von Entwick- lung und Intelligenz ehemaliger Frühgeborener nun um den Zeitraum des "Zufrühgeborenseins" korrigiert werden? Und falls ja - bis zu welchem Alter? Eine niederländische Arbeitsgruppe kam in einer aktuellen Ausgabe des Journals of Pediatrics zu einem Ergebis.}

$F_{\text {ind }}^{\text {in }}$ ir die Untersuchung wurden 275 ehemalige Frühgeborene von $24+5$ bis

$29+6$ Schwangerschaftswochen im korrigierten Alter von gut 5 Jahren einer umfassenden Intelligenztestung unterzogen. Anschließend wurde verglichen, ob auch in diesem Alter noch ein relevanter Unterschied im Ergebnis besteht, wenn die Alterskorrektur "eingerechnet" wird. Die Differenz schwankte in den Untertests zwischen 0-15 IQ-Punkten. Wenig überraschend waren auch in diesem Alter alle korrigierten IQ-Scores signifikant höher als die unkorrigierten $(\mathrm{p}<0,001)$. van Veen S et al. Consequences of correcting intelligence quotient for prematurity at age 5

\section{Kommentar}

Das Ergebnis dieser Untersuchung ist plausibel. Lenkt es doch die Aufmerksamkeit auf eine besondere Patientengruppe mit zum Teil zahlreichen Langzeitproblemen. Wir müssen uns klarmachen, dass in der Studie eine Gruppe von Menschen mit einem Geburtsgewicht zwischen 550 und $1.680 \mathrm{~g}$, einer Rate an bronchopulmonalen Dysplasien von $16 \%$ und Hirnblutungen von Grad III und mehr von $4 \%$ präsent sind. Das entspricht so years. J Pediatr 2016;173:90-5

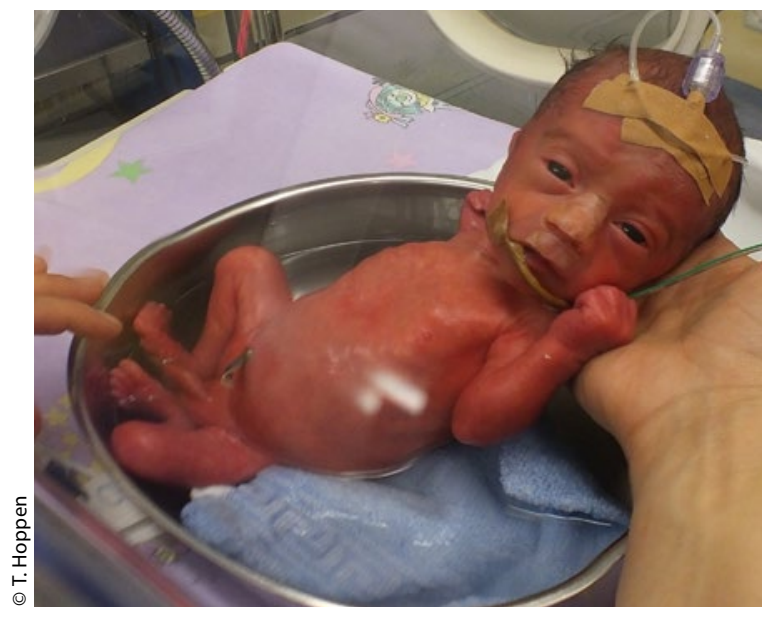

Baden im Inkubator - eine schöne Seite des „Frühchen-Daseins" auf Station gar nicht dem Bevölkerungsdurchschnitt. Die American Academy of Pediatrics empfiehlt eine Alterskorrektur ehemaliger Frühgeborener innerhalb der ersten drei Lebensjahre. Dies beruht auf der Annahme, dass in einem höheren Alter die Differenz zwischen korrigiertem und unkorrigiertem Alter in Bezug auf IQ-Ergebnisse keine Signifikanz mehr zeige und somit vernachlässigbar sei. Van Veen et al. regen nun mit Recht an, dies zu überdenken. Fairerweise sollten demnach in jedem Fall zeitgleich individuelle korrigierte und unkorrigierte Scores erhoben werden. So wäre jeweils eine bessere Grundlage zur individuellen Beurteilung und gezielten Langzeitförderung vorhanden. Das hat Gewicht!

Offensichtlich besteht eine Diskrepanz durch die zeitlichen und komorbiditätsbedingten Einflüsse für das ehemalige einzelne Frühgeborene möglicherweise ein Leben lang. Für die Beachtung der Alterskorrektur ehemaliger Frühgeborener bedarf es somit eines geschärften Bewusstseins bei den professionellen Beurteilern wie beispielsweise bei den Personen, die die Schuleingangsuntersuchung durchführen, aber auch generell in der gesamten Bevölkerung. Diese Erkenntnis darf nicht nur dem Forscher vertraut sein. Im Editorial des Journal of Pediatrics derselben Ausgabe [Doyle LW et Anderson PJ. J Pediatr 2016;173:11-2] wird ausdrücklich betont: Die Alterskorrektur bei Frühgeborenen sollte für Behandler und Familien in einem Gesamtkontext gesehen werden und ist deshalb ganz wesentlich davon abhängig zu machen, ob das Kind davon profitiert oder nicht. Da die Zahl überlebender extremer Frühgeborener weiter zunehmen wird, bedarf es für diese Fragestellung international anerkannter Standards.

Dr. Thomas Hoppen 